\title{
Effect of pulmonary exacerbations on long- term lung function decline in cystic fibrosis
}

\author{
Valerie Waters***, Sanja Stanojevic ${ }^{\star * *}$, Eshetu G. Atenafu", Annie Lu*, \\ Yvonne $\mathrm{Yau}^{+}$, Elizabeth Tullis ${ }^{\S}$ and Felix Ratjen ${ }^{\dagger}$
}

ABSTRACT: It is unknown what proportion of long-term lung function decline in cystic fibrosis (CF) is explained by pulmonary exacerbations. The aim of this study was to determine how exacerbations requiring hospitalisation contribute to the course of CF lung disease.

This was a retrospective cohort study. The primary outcome was the rate of decline of forced expiratory volume in $1 \mathrm{~s}\left(\mathrm{FEV}_{1}\right) \%$ predicted.

Out of 851 subjects, $415(48.8 \%)$ subjects had $\geqslant 1$ exacerbation. After adjustment for confounders, the annual rate of FEV 1 decline in those without an exacerbation was $1.2 \%$ per $\mathrm{yr}$ (95\% Cl 1.0-1.5), compared with $2.5 \%$ per yr (95\% Cl 2.1-2.8) in those with an exacerbation. The proportion of overall FEV 1 decline associated with $\geqslant 1$ exacerbation was $52 \%(95 \% \mathrm{Cl} 35.0-68.9)$. For a given number of exacerbations, the annual rate of $\mathrm{FEV} 1$ decline was greatest in subjects with $\leqslant 6$ months between exacerbations.

Half of FEV 1 decline seen in CF patients was associated with pulmonary exacerbations. Time between exacerbations, specifically $\leqslant 6$ months between exacerbations, plays an important contribution to overall lung function decline. These findings support using time to next exacerbation as a clinical end-point for CF trials.

KEYWORDS: Forced expiratory volume in $1 \mathrm{~s}$, cystic fibrosis, pulmonary exacerbation

D) ulmonary exacerbations are significant clinical events in the lives of cystic fibrosis (CF) patients. In 2007, 38\% of all CF patients in the USA were treated with intravenous antibiotics for $\geqslant 1$ pulmonary exacerbation [1]. Although there is controversy in how to define a pulmonary exacerbation, an increased number of exacerbations is clearly associated with increased morbidity and mortality in CF $[2,3]$. Several studies have shown that more pulmonary exacerbations are associated with a steeper subsequent decline in lung function, specifically forced expiratory volume in $1 \mathrm{~s}$ (FEV1), particularly among children [4-6]. Multiple datasets, including those from the Toronto CF Database housed at the Hospital for Sick Children (Toronto, ON, Canada), have examined the effect of pulmonary exacerbations, as discrete events, on lung function and have demonstrated that up to one-third of $\mathrm{CF}$ patients will not recover their baseline FEV1, with an $\sim 3 \%$ decrease in FEV1 following an exacerbation [7-10]. These studies also identify risk factors for failing to recover lung function following a pulmonary exacerbation, including greater initial drops in FEV1. However, despite this knowledge, we still do not understand how repeated pulmonary exacerbations affect lung function over the lifetime of a CF patient. This knowledge is crucial in determining how important it is to prevent pulmonary exacerbations, which are often measured as study outcomes in clinical trials in CF. Therefore, the aim of this study was to model FEV1 decline in a paediatric and adult CF population over a 12-yr period and determine the role of pulmonary exacerbations (requiring hospital admission for i.v. antibiotics) in that decline.

\section{MATERIALS AND METHODS}

\section{Study design and patient population}

This was a retrospective cohort study of subjects with CF followed at the Hospital for Sick Children and St Michael's Hospital (Toronto, ON, Canada) from 1997 to 2008. The primary outcome was the annual rate of decline in FEV1 \% predicted.

Subjects were included in the study if they had a confirmed diagnosis of CF based on: 1) the presence of clinical features consistent with $\mathrm{CF}$; or 2) a positive family history for $\mathrm{CF}$, and $>60 \mathrm{mEq} \cdot \mathrm{L}^{-1}$ measured by quantitative pilocarpine iontophoresis test, genetic testing showing two CF-causing mutations or a nasal potential difference consistent with CF [11]. Subjects were excluded if they were unable to perform reproducible spirometry. There were no subjects in this either two documented sweat chloride values
AFFILIATIONS

*Division of Infectious Diseases,

${ }^{f}$ Division of Respiratory Medicine, Dept of Pediatrics,

${ }^{*}$ Child Health Evaluative Sciences, ${ }^{+}$Division of Microbiology, Dept of Pediatric Laboratory Medicine, The Hospital for Sick Children,

"Dept of Biostatistics, Princess Margaret Hospital, University Health Network and

${ }^{\S}$ Division of Respirology and Keenan Research Centre of Li Ka Shing Knowledge Institute, Dept of Medicine, St. Michael's Hospital, University of Toronto, Toronto, ON, Canada.

**Both authors contributed equally to this work.

CORRESPONDENCE

V. Waters

Division of Infectious Diseases,

Hospital for Sick Children

555 University Avenue

Toronto

ON

M5G $1 \times 8$

Canada

E-mail: valerie.waters@sickkids.ca

Received:

Sept 132011

Accepted after revision:

Nov 042011

First published online:

Dec 012011 
cohort who were identified by newborn screening. Data were censored at lung transplantation or death.

This study was approved by the Research Ethics Board at the Hospital for Sick Children (1000013759) and St Michael's Hospital $\left(09-087^{\mathrm{C}}\right)$.

\section{Data collection and definitions}

The data for this study was extracted from the Toronto CF Database. This encounter-based database prospectively collects information from paediatric and adult CF subjects from every visit including sputum microbiology, medications and pulmonary function testing [12]. Specific information that was unavailable from the database was retrieved through systematic review of hospital health records by one of the study investigators (A. Lu).

A pulmonary exacerbation was defined as a hospitalisation for respiratory symptoms requiring antibiotics (96\% of patients were administered i.v. antibiotics), as previously described [10, 13]; hospitalisations for gastrointestinal complications were not included. Consecutive pulmonary exacerbations within 3 weeks of antibiotic treatment were considered as a single event [7]. Exacerbation history was analysed as ever/ never, total number during the study period (grouped as 0,1 , $2-4, \geqslant 5$ ) and as the minimum time between two consecutive exacerbations. FEV1 values were corrected for height, age and sex and analysed as \% predicted [14]. Body mass index (BMI) was categorised into normal, underweight and overweight. In children ( $<18$ yrs of age), BMI was first converted to centiles and categorised into normal, underweight and overweight based on standard cut-offs ( $<5$ th centile, 5-85th centile, $>85$ centile) [15]. In adults, we used standard cut-offs $<18,18-25$, $\geqslant 25 \mathrm{~kg} \cdot \mathrm{m}^{-2}$.

\section{Statistical analysis}

Baseline characteristics for the cohort were defined based on data from the first year of observation. The overall annual rate of FEV1 decline was estimated with all recorded measurements using generalised estimating equation (GEE) models, which adjust for the correlated nature of repeated measures in an individual. In the multivariable models, we adjusted for age (yrs), sex (male or female), genotype (homozygous $\Delta \mathrm{F} 508$, heterozygous $\Delta \mathrm{F} 508$, other, unknown), baseline FEV1 $\left(\mathrm{L} \cdot \mathrm{s}^{-1}\right)$, year of measurement, age at diagnosis ( $<1 \mathrm{yr},<12 \mathrm{yrs}, \leqslant 18 \mathrm{yrs}$, $>18$ yrs, unknown), BMI (normal, underweight, overweight), any previous Pseudomonas aeruginosa or Burkholderia cepacia complex (BCC) infection, a history of allergic bronchopulmonary aspergillosis (ABPA) (as defined by the treating physician in combination with increased immunoglobulin E levels) [10], pancreatic insufficiency (defined by enzyme usage) and CFrelated diabetes (CFRD) (based on oral glucose tolerance test). Age and BMI were treated as time-varying variables. Socioeconomic status was not available. Furthermore, Medicaid insurance was not investigated since Canada has universal healthcare insurance. Methicillin-resistant Staphylococcus aureus was not included due to its low prevalence in the CF population in Canada [16]. Factors which were marginally significant $(\mathrm{p}<0.15)$ using univariable analysis, or those with strong a priori hypothesis (e.g. sex, age) were entered into a multivariable model using a step-wise approach. Variables were maintained in the multivariable linear GEE model if they were independently associated with FEV1 decline, or if they changed the coefficient for the effect of exacerbation on slope on FEV1. Effect modifications by exacerbations (ever versus never) and age ( $>18$ versus $\leqslant 18$ yrs) was evaluated by testing for interactions in the model and conducting stratified analyses where appropriate. In addition, sensitivity analysis was performed excluding subjects $>50$ yrs of age, subjects with a history of BCC infection and subjects with ABPA. The extent to which exacerbations contribute to overall lung function decline over time was estimated using the formula $\left(\beta_{0}-\beta_{1}\right) / \beta_{0}$, where $\beta_{0}$ is the time coefficient in the model without the exacerbation covariate and $\beta_{1}$ is the slope in the model which included the exacerbation covariate and potential confounding variables [17].

\section{RESULTS}

\section{Study population characteristics}

From 1997 to 2008, 851 subjects with CF were followed for a median (interquartile range) of 6.7 (2.4-9.7) yrs. There were 1,882 pulmonary exacerbations during the study period. A total of $415(48.8 \%)$ subjects had at least one pulmonary exacerbation requiring hospitalisation and antibiotics. The median number of exacerbations per subject was 3 (range 119) and the median number of exacerbations per subject per year was 2 (range 1-5). The average time between two consecutive exacerbations was 1 yr (range 2 months-6 yrs).

Compared with subjects who had no pulmonary exacerbations during the study period, subjects with $\geqslant 1$ pulmonary exacerbation were followed for longer; were younger at baseline; were more likely to be female; were diagnosed at a younger age; were more likely to be homozygous for $\Delta \mathrm{F} 508$; to be pancreatic insufficient; to be underweight; to have CFRD; to have had $P$. aeruginosa or BCC infection; to have a history of $\mathrm{ABPA}$ and to have lower baseline FEV1 (table 1).

\section{Factors influencing FEV1 decline}

Using univariable analysis, the overall rate of FEV1 \% predicted decline for the entire study population was $1.6 \%$ per yr (95\% CI 1.6-1.7). In subjects with $\geqslant 1$ pulmonary exacerbation, the annual rate of decline was $1.8 \%$ per yr $(95 \%$ CI 1.8-1.9) compared with $1.1 \%$ per yr (95\% CI $1.0-1.1)$ in subjects without any exacerbations. These rates of FEV1 decline were similar in children and adults; younger subjects had a higher baseline FEV1 (fig. 1).

In the multivariable analysis, the annual rate of decline in FEV1 was modified by exacerbation history (interaction $\mathrm{p}<0.0001$ ) such that the rate of decline was steeper in the exacerbation group. In addition, the rate of FEV1 decline was proportionally greater in older subjects, those with an earlier study entry, lower baseline FEV1, underweight subjects and those with a history of BCC infection (table 2). Although significant in the univariable analysis, $P$. aeruginosa infection, pancreatic insufficiency and CFRD did not contribute to the final multivariable model. After adjusting for potential confounders, the rate of FEV1 decline in subjects with $\geqslant 1$ exacerbation was $2.5 \%$ per yr (95\% CI $2.1-2.8$ ) compared with $1.2 \%$ per yr (95\% CI $1.0-1.5)$ in subjects without an exacerbation. The proportion of the overall FEV1 decline $(-1.62 \%$ per yr) associated with pulmonary exacerbations $(-2.47 \%$ per yr) was $52 \%(95 \% \mathrm{CI}$ 35.0-68.9). 
TABLE 1 Study population characteristics at baseline

\begin{tabular}{|c|c|c|c|}
\hline Variable & No exacerbations & $\geqslant 1$ exacerbation & Total \\
\hline Subjects $n$ & 436 & 415 & 851 \\
\hline Female & $176(40.4)$ & $205(49.4)$ & $381(44.8)$ \\
\hline Age yrs & $19.6(9.6-31.1)$ & $16.0(8.5-25.9)$ & $18.0(8.7-28.5)$ \\
\hline \multicolumn{4}{|l|}{ Age at diagnosis yrs } \\
\hline$\leqslant 18$ & $22(5.1)$ & $21(5.1)$ & $43(5.1)$ \\
\hline$>18$ & $69(15.8)$ & $23(5.5)$ & $92(10.8)$ \\
\hline Unknown & $1(0.2)$ & $1(0.2)$ & $2(0.2)$ \\
\hline \multicolumn{4}{|l|}{ Genotype } \\
\hline Homozygous $\Delta \mathrm{F} 508$ & $160(36.7)$ & $189(45.5)$ & $349(41.0)$ \\
\hline \multicolumn{4}{|l|}{ Body mass index } \\
\hline Underweight & $23(5.3)$ & $30(7.2)$ & $53(6.2)$ \\
\hline Normal weight & 321 (73.6) & $346(83.4)$ & 667 (78.4) \\
\hline Overweight & $92(21.1)$ & $39(9.4)$ & $131(15.4)$ \\
\hline Cystic fibrosis-related diabetes mellitus & $30(6.9)$ & $38(9.1)$ & $68(8.0)$ \\
\hline Pseudomonas aeruginosa infection & $197(45.2)$ & $270(65.1)$ & 467 (54.9) \\
\hline Burkholderia cepacia complex infection & $62(14.2)$ & $71(17.1)$ & $133(15.6)$ \\
\hline Allergic bronchopulmonary aspergillosis & $0(0)$ & $46(11.1)$ & $46(5.4)$ \\
\hline \multicolumn{4}{|l|}{ Lung Function } \\
\hline Average number of pulmonary function tests per yr per subject & $1(1-2)$ & $2(1-3)$ & $1(1-2)$ \\
\hline Baseline FEV $1 \%$ pred & $74.8 \pm 26.7$ & $64.0 \pm 24.5$ & $69.5 \pm 26.2$ \\
\hline
\end{tabular}

Data are presented as median (interquartile range), $\mathrm{n}(\%)$ or mean $\pm \mathrm{SD}$, unless otherwise stated. FEV 1 : forced expiratory volume in $1 \mathrm{~s} ; \%$ pred: \% predicted.

\section{Sensitivity analyses}

Excluding subjects $>50$ yrs of age, those with a history of ABPA or those who subsequently had a transplant did not

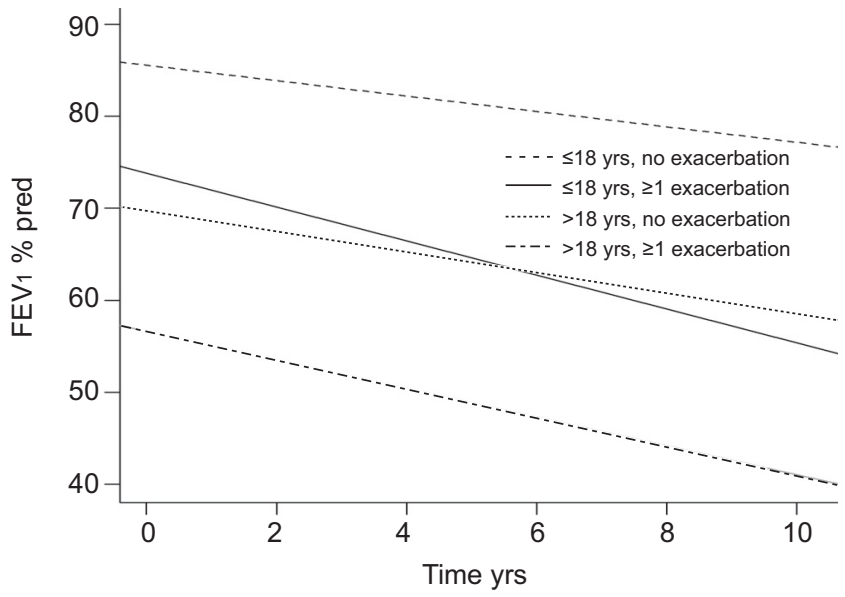

FIGURE 1. Forced expiratory volume in $1 \mathrm{~s}\left(\mathrm{FEV}_{1}\right) \%$ predicted decline over time in subjects aged either $\leqslant 18 \mathrm{yrs}$ or $>18 \mathrm{yrs}$ and either with or without exacerbation change the interpretation of the results. When we limited the analysis to those without a history of infection with BCC, the adjusted slope of FEV1 decline was slightly attenuated $(-2.0 \%$ per $\mathrm{yr}, 95 \% \mathrm{CI}-2.2--1.7)$. After adjusting for potential confounders, the annual rate of decline was the same in both children and adults.

\section{The effect of number and timing of pulmonary exacerbations on FEV1 decline}

After adjusting for potential confounders, the total number of exacerbations (grouped as $0,1,2-4, \geqslant 5$ over the study period) was a significant predictor of annual FEV1 decline. The annual rate of FEV1 decline for each exacerbation group was compared with the rate of FEV1 decline in the group with no exacerbations and the relative difference is represented in figure 2. The rate of FEV1 decline for the group with only one exacerbation during the study period did not differ significantly from the group with no exacerbations $(0.7 \%, 95 \% \mathrm{CI}$ -1.3-2.5). Relative to the group with no exacerbations, the slope for FEV1 decline was $-5.3 \%$ (95\% CI $-7.0--3.5)$ lower for the group with $2-4$ exacerbations and $-7.9 \%$ (95\% CI $-9.6--6.1)$ lower for those with $\geqslant 5$ exacerbations over the study period.

Subsequently, we further categorised those patients with $>2$ exacerbations over the study period based on the minimum 


\begin{tabular}{|c|c|c|c|c|}
\hline Variable & \multicolumn{2}{|c|}{ No exacerbations } & \multicolumn{2}{|c|}{$\geqslant 1$ exacerbation } \\
\hline Longer duration of follow up yrs & $-1.21(-1.48--0.95)$ & $<0.0001$ & $-2.43(-2.78--2.08)$ & $<0.0001$ \\
\hline Increasing age yrs & $-0.19(-0.26--0.13)$ & $<0.0001$ & $-0.25(-0.35--0.16)$ & $<0.0001$ \\
\hline Lower baseline FEV $1 \%$ pred & $-0.81(-0.84--0.78)$ & $<0.0001$ & $-0.73(-0.78--0.69)$ & $<0.0001$ \\
\hline \multicolumn{5}{|l|}{ BMI } \\
\hline Normal weight & Ref. & Ref. & Ref. & Ref. \\
\hline Underweight & $-3.56(-4.85--2.27)$ & $<0.0001$ & $-8.00(-8.73--7.26)$ & $<0.0001$ \\
\hline Overweight & $3.81(2.90-4.72)$ & $<0.0001$ & $4.21(3.35-5.09)$ & $<0.0001$ \\
\hline BCC infection & $-6.46(-8.80--4.12)$ & $<0.0001$ & $-2.78(-5.53--0.08)$ & $<0.0001$ \\
\hline
\end{tabular}

After adjusting for the other potential confounders, patients in the no exacerbations group with Burkholderia cepacia complex (BCC) infection have an annual rate of forced expiratory volume in $1 \mathrm{~s}\left(\mathrm{FEV}_{1}\right)$ decline that is $6.46 \%$ greater than the group without BCC infection. \% pred: \% predicted; BMI: body mass index; Ref.: reference.

time between any two consecutive exacerbations ( $\leqslant 6$ months, $>6$ months). Relative to the group with no exacerbations, the greatest FEV1 decline was observed when the time between two consecutive exacerbations was $<6$ months (fig. 3 ); this observation was consistent regardless of the total number of exacerbations during the study period. Subjects with the same total number of exacerbations over the 12 yrs (2-4 exacerbations), but $<6$ months between exacerbations had a greater FEV1 decline $(-6.9 \%$ (95\% CI -9.5- -4.2)) compared with the group with $>6$ months between exacerbation $(-4.4 \%$ (95\% CI $-6.43--2.40)$ ) (fig. 3a). For subjects with $\geqslant 5$ exacerbations and a shorter interval between subsequent exacerbations, the FEV1

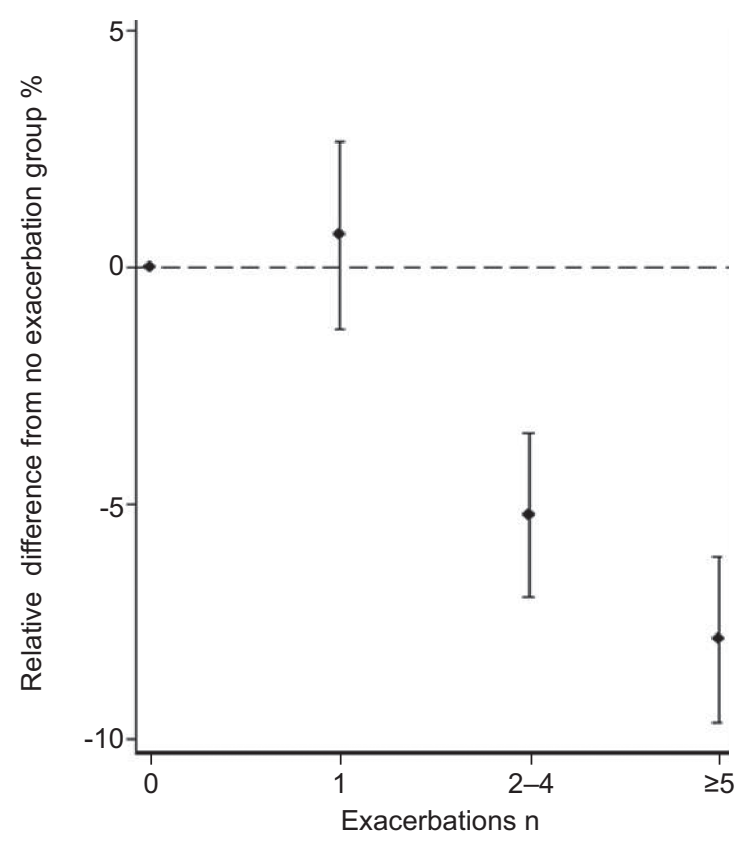

FIGURE 2. The relative per cent difference in the rate of forced expiratory volume in $1 \mathrm{~s}$ decline (with 95\% confidence intervals) for each group according to the number of pulmonary exacerbations during the study period, compared to the group with no pulmonary exacerbations. decline was $-8.1 \%$ (95\% CI -9.9- -6.3) lower compared with the group with no exacerbations, compared with -6.4 (95\% CI 10.5- -2.3) in the group with $>6$ months between exacerbations (fig. 3b).

\section{DISCUSSION}

To our knowledge, this is the first study to quantify the effect of pulmonary exacerbations on long-term lung function decline in $\mathrm{CF}$ and to show that half of FEV1 decline in $\mathrm{CF}$ patients is associated with severe pulmonary exacerbations requiring hospitalisation and i.v. antibiotics. In addition, we confirm that more exacerbations are associated with greater FEV1 decline, and for the first time demonstrate that time between consecutive exacerbations also plays an important part in long-term lung function decline.

Within the spectrum of illness that can be considered a pulmonary exacerbation, severe pulmonary exacerbations requiring i.v. antibiotics has clearly been shown to be associated with lung function decline $[4,6]$, such that a quarter of adult and paediatric CF patients do not recover their baseline FEV1 $[7,8]$. The annual rate of decline observed in our CF population is consistent with other studies [18]. However, few studies actually quantify what proportion of FEV1 decline in $\mathrm{CF}$ was associated with such exacerbations over time. AMADORI et al. [19] followed the FEV1 decline of 51 adult CF patients over 5 yrs and reported an FEV1 loss of $\sim 30 \mathrm{~mL}$ for each exacerbation episode. The study was limited, however, by a small sample size and FEV1 decline reported in $\mathrm{mL}$, which is difficult to interpret since lung function is strongly related to body size and, therefore, commonly interpreted as \% predicted in clinical settings. In comparison, our study followed almost $1,000 \mathrm{CF}$ patients over a 12 -yr period, the longest reported follow up for FEV1 decline, and we report that half of FEV1 decline in CF was associated with pulmonary exacerbations. Although we compared differences in rates of FEV1 decline, these findings are still clinically relevant as the most severely affected subjects $(\geqslant 5$ exacerbations, $\leqslant 6$ months between exacerbations) had double the rate of FEV1 decline ( $\sim 2 \%$ pred per yr) compared with subjects without any exacerbations during the study period $(\sim 1 \%$ pred per yr). These results 

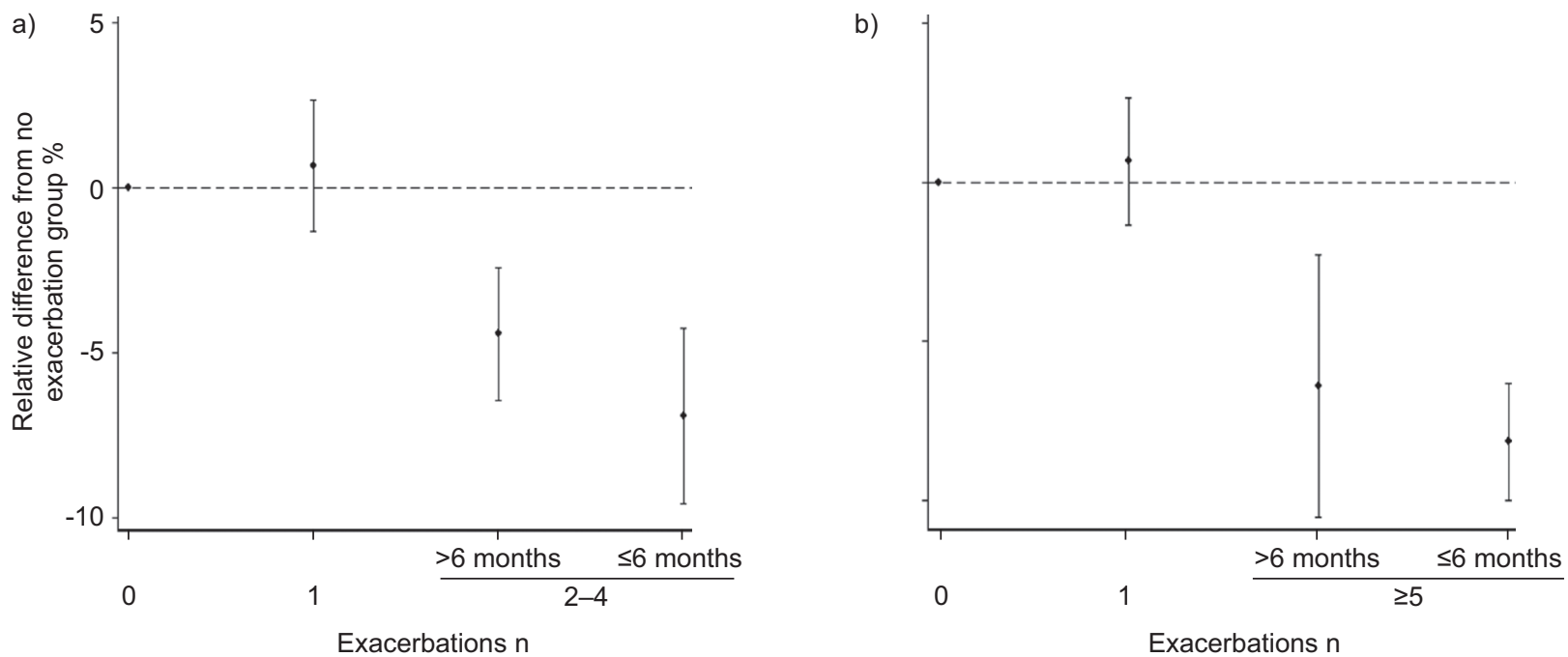

FIGURE 3. The relative per cent difference in the rate of forced expiratory volume in $1 \mathrm{~s}$ decline (with $95 \%$ confidence intervals) compared with the group with no pulmonary exacerbations according to time between exacerbations (>6 months or $\leqslant 6$ months) for subjects with a) $2-4$ exacerbations during the study period and b) $\geqslant 5$ exacerbations during the study period.

further emphasise the important contribution exacerbations have on disease progression in patients with $\mathrm{CF}$.

This study confirmed that an increasing number of pulmonary exacerbations were associated with greater FEV1 decline. SANDERS and co-workers [4, 7] showed that any exacerbation in children with $\mathrm{CF}$ and $\geqslant 3$ exacerbations in a single year in adults with CF were associated with a significantly increased rate of FEV1 decline and that those who failed to recover to baseline lung function following an exacerbation were more likely to have a subsequent pulmonary exacerbation in the 3,6, and 12 months following treatment. DE BOER et al. [20] also found that adult $\mathrm{CF}$ patients with $\geqslant 3$ exacerbations per year were at increased risk of a 5\% decline in baseline FEV1, although their definition of an exacerbation included patients who were treated with either oral or i.v. antibiotics. These studies suggest that it is not only the frequency of exacerbations but it is also repetitive, consecutive exacerbations within a short time period that contribute to lung function decline in $\mathrm{CF}$. These findings further support that patients with $\mathrm{CF}$ would benefit from close monitoring following an exacerbation. While our study agrees with these observations, in contrast, we do not treat exacerbations as discrete events and therefore describe the contribution of exacerbations to longterm lung function decline over a period of $12 \mathrm{yrs}$.

In our study we examined the effect of time between exacerbations and show for the first time that two exacerbations within 6 months contribute towards a faster rate of lung function decline. This is consistent with previous studies that found 3 exacerbations per year represent a crucial tipping point in terms of CF lung function decline, as the minimum time between exacerbations in this instance has to be within 6 months $[4,20]$. Although rate of pulmonary exacerbations and time between pulmonary exacerbations are clearly correlated, they are not necessarily equivalent. These data contribute to our understanding of the relevance of consecutive exacerbations and have important implications for clinical research.
Pulmonary exacerbations are frequently used as a study outcome measure in CF trials, and are usually measured as the risk of a pulmonary exacerbation during the study period [21-23]. Our findings suggest that it is not a pulmonary exacerbation per se, but consecutive exacerbations within a narrow time period, measured in this case by minimum time between exacerbations, which strongly impact the rate of lung function decline. This has two important implications for $\mathrm{CF}$ intervention trials. First, preventing an exacerbation is more important in patients who have recently had an exacerbation than in patients whose prior exacerbation is more remote. Thus, history of exacerbation and, more specifically, the time since last exacerbation, may be an important inclusion criterion for trials. Secondly, when comparing a treatment and a placebo group in an interventional study, the two groups should be balanced with respect to pulmonary exacerbation history as this may influence the response to therapy and could potentially bias interpretation. For example, in the randomised, double-blind, placebo-controlled trial of high-dose ibuprofen in patients with $\mathrm{CF}$, the treatment group had a significantly slower annual decline in FEV1 than the placebo groups despite the fact that there was no significant difference between the groups in the number of hospitalisations for pulmonary exacerbations during the study period [24]. If there were more subjects with recent exacerbations prior to study entry in the treatment group than in the placebo group, preventing a pulmonary exacerbation during the study period may have had a more significant impact on lung function decline in the treated subjects than in the untreated subjects. Thus, although the number of exacerbations was not different between the two groups, the time between exacerbations may have been extended by high-dose ibuprofen therapy. Our findings have implications for future clinical trials that evaluate lung function decline as the primary outcome. Enrolling patients with a history of recent exacerbation may result in a more powerful study design, requiring shorter follow-up time and smaller sample sizes to show therapeutic benefit. 


\section{Strengths and limitations}

To our knowledge, this study reports the longest follow-up of lung function decline in both paediatric and adult patients with CF, with over 20,323 observations in 851 subjects over a $12-y r$ period. While this study does not examine the factors associated with lung function decline for each discrete exacerbation as previously performed $[7,10]$, it is the first to detail the overall effect of exacerbations on CF lung function decline over time. Another potential limitation is our definition of pulmonary exacerbation. In this study, we defined a pulmonary exacerbation as a hospitalisation for respiratory symptoms requiring antibiotics $(96 \%$ of patients received i.v. antibiotics). By definition, this includes only the more severe pulmonary exacerbations in the analysis; it is unclear what the role of milder exacerbations is in CF pulmonary deterioration. However, it is a clinically relevant definition. Previous publications have demonstrated that pulmonary exacerbations requiring i.v. antibiotics are associated with significant pulmonary inflammation and it is thus likely that the greatest effect on CF lung function decline would be seen in this patient population [25]. Finally, this study was retrospective which may limit the amount of information that can be obtained about respiratory symptoms at the time of an exacerbation. Even with a prospective study design it is difficult to infer causality and whether FEV1 decline occurred prior to the identification of the exacerbation or was a direct result of the exacerbation [4, 21, 22, 26]. Unlike previous studies where exacerbations were treated as discrete events, we examined the contribution of exacerbations on overall lung function decline.

\section{Conclusions}

In conclusion, one half of the FEV1 decline seen in CF patients was associated with severe pulmonary exacerbations requiring hospitalisation and antibiotics. In addition, we demonstrated that the time between exacerbations, specifically $\leqslant 6$ months between exacerbations, is an important predictor of overall lung function decline. These findings support using time to next exacerbation as a clinical end-point for CF trials.

\section{SUPPORT STATEMENT}

This work was supported by the Canadian Cystic Fibrosis Foundation.

\section{STATEMENT OF INTEREST}

None declared.

\section{REFERENCES}

1 Flume PA, Mogayzel PJ Jr, Robinson KA, et al. Cystic fibrosis pulmonary guidelines: treatment of pulmonary exacerbations. Am J Respir Crit Care Med 2009; 180: 802-808.

2 Marshall BC. Pulmonary exacerbations in cystic fibrosis: it's time to be explicit! Am J Respir Crit Care Med 2004; 169: 781-782.

3 Liou TG, Adler FR, Fitzsimmons SC, et al. Predictive 5-year survivorship model of cystic fibrosis. Am J Epidemiol 2001; 153: 345-352.

4 Sanders DB, Bittner RC, Rosenfeld M, et al. Pulmonary exacerbations are associated with subsequent FEV1 decline in both adults and children with cystic fibrosis. Pediatr Pulmonol 2011; 46: 393-400.

5 Emerson J, Rosenfeld M, McNamara S, et al. Pseudomonas aeruginosa and other predictors of mortality and morbidity in young children with cystic fibrosis. Pediatr Pulmonol 2002; 34: 91-100.
6 Konstan MW, Morgan WJ, Butler SM, et al. Risk factors for rate of decline in forced expiratory volume in one second in children and adolescents with cystic fibrosis. J Pediatr 2007; 151: 134-139.

7 Sanders DB, Bittner RC, Rosenfeld M, et al. Failure to recover to baseline pulmonary function after cystic fibrosis pulmonary exacerbation. Am J Respir Crit Care Med 2010; 182: 627-632.

8 Sanders DB, Hoffman LR, Emerson J, et al. Return of FEV1 after pulmonary exacerbation in children with cystic fibrosis. Pediatr Pulmonol 2010; 45: 127-134.

9 Collaco JM, Green DM, Cutting GR, et al. Location and duration of treatment of cystic fibrosis respiratory exacerbations do not affect outcomes. Am J Respir Crit Care Med 2010; 182: 1137-1143.

10 Waters V, Atenafu EG, Salazar JG, et al. Chronic Stenotrophomonas maltophilia infection and exacerbation outcomes in cystic fibrosis. J Cyst Fibros 2012; 11: 8-13.

11 Farrell PM, Rosenstein BJ, White TB, et al. Guidelines for diagnosis of cystic fibrosis in newborns through older adults: Cystic Fibrosis Foundation consensus report. J Pediatr 2008; 153: S4-S14.

12 Kerem E, Reisman J, Corey M, et al. Prediction of mortality in patients with cystic fibrosis. N Engl J Med 1992; 326: 1187-1191.

13 Waters V, Yau Y, Prasad S, et al. Stenotrophomonas maltophilia in cystic fibrosis: serologic response and effect on lung disease. Am J Respir Crit Care Med 2011; 183: 635-640.

14 Stanojevic S, Wade A, Cole TJ, et al. Spirometry centile charts for young Caucasian children: the Asthma UK Collaborative Initiative. Am J Respir Crit Care Med 2009; 180: 547-552.

15 Kuczmarski RJ, Ogden CL, Guo SS, et al. 2000 CDC Growth Charts for the United States: methods and development. Vital Health Stat 2002; 246: 1-190.

16 Cystic Fibrosis Canada. Canadian Patient Data Registry Report, 2009. www.cysticfibrosis.ca/en/publications/CanadianCFPatient Statistics.php. Date last updated: July 18, 2011. Date last accessed: October 24, 2011.

17 Hardoon SL, Morris RW, Whincup PH, et al. Rising adiposity curbing decline in the incidence of myocardial infarction: 20-year follow-up of British men and women in the Whitehall II cohort. Eur Heart J 2012; 33: 478-485.

18 Que C, Cullinan P, Geddes D. Improving rate of decline of FEV1 in young adults with cystic fibrosis. Thorax 2006; 61: 155-157.

19 Amadori A, Antonelli A, Balteri I, et al. Recurrent exacerbations affect FEV1 decline in adult patients with cystic fibrosis. Respir Med 2009; 103: 407-413.

20 de Boer K, Vandemheen KL, Tullis E, et al. Exacerbation frequency and clinical outcomes in adult patients with cystic fibrosis. Thorax 2011; 66: 680-685.

21 Ramsey BW, Pepe MS, Quan JM, et al. Intermittent administration of inhaled tobramycin in patients with cystic fibrosis. Cystic Fibrosis Inhaled Tobramycin Study Group. N Engl J Med 1999; 340: 23-30.

22 Fuchs HJ, Borowitz DS, Christiansen DH, et al. Effect of aerosolized recombinant human DNase on exacerbations of respiratory symptoms and on pulmonary function in patients with cystic fibrosis. The Pulmozyme Study Group. N Engl J Med 1994; 331: 637-642.

23 Saiman L, Marshall BC, Mayer-Hamblett N, et al. Azithromycin in patients with cystic fibrosis chronically infected with Pseudomonas aeruginosa: a randomized controlled trial. JAMA 2003; 290: 1749-1756.

24 Konstan MW, Byard PJ, Hoppel CL, et al. Effect of high-dose ibuprofen in patients with cystic fibrosis. N Engl J Med 1995; 332: 848-854.

25 Ordonez CL, Henig NR, Mayer-Hamblett N, et al. Inflammatory and microbiologic markers in induced sputum after intravenous antibiotics in cystic fibrosis. Am J Respir Crit Care Med 2003; 168: 1471-1475.

26 Rosenfeld M, Emerson J, Williams-Warren J, et al. Defining a pulmonary exacerbation in cystic fibrosis. J Pediatr 2001; 139: 359-365. 